\title{
Globe
}

Revue internationale d'études québécoises

\section{Scène contemporaine et recherches interdisciplinaires au Québec}

\section{Marie-Christine Lesage}

Volume 11, numéro 2, 2008

URI : https://id.erudit.org/iderudit/1000527ar

DOI : https://doi.org/10.7202/1000527ar

Aller au sommaire du numéro

Éditeur(s)

Globe, Revue internationale d'études québécoises

ISSN

1481-5869 (imprimé)

1923-8231 (numérique)

Découvrir la revue

Citer cette note

Lesage, M.-C. (2008). Scène contemporaine et recherches interdisciplinaires au Québec. Globe, 11(2), 169-184. https://doi.org/10.7202/1000527ar d'utilisation que vous pouvez consulter en ligne.

https://apropos.erudit.org/fr/usagers/politique-dutilisation/ 


\section{NOTE CRITIQUE}

\section{SCENNE CONTEMPORAINE \\ ET RECHERCHES \\ INTERDISCIPLINAIRES \\ AU QUÉBEC}

\section{MARIE-CHRISTINE LESAGE}

Centre des auteurs dramatiques (CEAD), Université du Québec à Montréal

$4+4+4+4+4+4+4+4+4+4+4+4+$

La scène contemporaine québécoise, à l'instar de la scène internationale, offre une diversité de formes que l'on peine de plus en plus à enclore dans un seul champ artistique: théâtre, danse, performance, arts médiatiques, installation, musique, etc. sont convoqués, selon des configurations diverses, pour donner naissance à des créations scéniques que l'on nomme souvent "hybrides", parce qu'elles échappent aux traditionnelles catégorisations en termes de disciplines artistiques. La liste est longue des formes artistiques contemporaines qui font interagir plusieurs arts, créant ainsi des œuvres protéiformes: théâtre-danse de Paula de Vasconcelos, danse théâtralisante et performative de Benoît Lachambre ou de Dave Saint-Pierre, installation-théâtre vidéographique de Denis Marleau, théâtre technologique de Robert Lepage, théâtre d'objets du Théâtre de la Pire Espèce, sans compter la pluralité des formes circassiennes et performatives, qui font appel à plusieurs arts. Devant la diversité grandissante de ces migrations artistiques, ou, pour le dire autrement, devant la présence accrue d'œuvres interartistiques sur les scènes québécoises, on ne peut que s'interroger sur le discours 
qui entoure ces créations, et plus particulièrement sur la recherche dont l'objet serait justement le décloisonnement des disciplines artistiques et son corollaire, des formes scéniques hétérogènes.

Si les recherches sur la scène contemporaine sont assez bien développées par secteur (théâtre contemporain, danse contemporaine, performance actuelle, nouveau cirque, etc.), force est de constater que peu d'études ont à ce jour attaqué de front la question des pratiques interartistiques. Étant donné l'intrication de différents langages artistiques sur la scène contemporaine, le travail théorique d'interprétation (dégager ses stratégies esthétiques, sa portée critique, l'objet de la représentation, etc.) appelle une réflexion de nature interdisciplinaire, qui est exigeante si l'on veut éviter les confusions notionnelles et les transferts conceptuels approximatifs. Il existe du côté des sciences et des sciences sociales des approches interdisciplinaires bien ancrées - c'est d'ailleurs dans ces secteurs que l'interdisciplinarité est apparue, pour faire contrepoids à l'hyperspécialisation des différents domaines, laquelle ne permettait plus d'approcher de nouveaux phénomènes culturels, sociaux, scientifiques présentant un degré accru de complexité, qui exigeaient une mise en commun des savoirs. La pensée du chercheur français Edgar Morin est à cet égard représentative de ce nouveau courant de pensée dans les sciences sociales'. Dans le domaine des arts de la scène, les performance studies américaines ont développé une réflexion plutôt sociologisante axée sur les identités culturelles, sexuelles, nationales. En Europe, la réflexion tend à repartir, à tort ou à raison, de la notion wagnérienne de Gesamtkunstwerk, voyant dans le mélange actuel des arts un héritage direct de l'œuvre d'art totale; pour certains, l'opéra serait aujourd'hui la forme interartistique par excellence, qui permettrait la rencontre du théâtre, du chant, de la musique, de la danse et de la vidéo. La recherche sur les pratiques interartistiques au Québec tend à osciller entre ces deux approches, selon le champ disciplinaire à partir duquel est initié le discours.

On retrouve peu d'ouvrages qui abordent ces formes scéniques de manière transversale, c'est-à-dire en discutant du mélange des arts comme des disciplines, sur le plan tant esthétique que théorique, voire épistémologique (ce dont une réflexion synthétique sur la scène contemporaine ne pourrait, me semble-t-il, faire l'économie). On retrouvera parfois un chapitre à l'inté-

$$
+4
$$

1. Pour une synthèse incroductive à la pensée de Morin, voir Introduction à la pensér complexe, Paris, Éditıons du Seuil, 2005. 
rieur d'un ouvrage collectif sur le theâtre québécois². Le champ thêâtral a vu émerger une scène accordant une place accrue aux autres arts comme le nouveau cirque, la musique ou encore le corps dansant, ce dont certaines revues se sont fait l'écho. Ainsi, les Cahiers de théâtre Jeu et L'Annuaire théatral présentent ponctuellement des dossiers nourris sur le sujet ${ }^{3}$. La revue Intermédialités, quant à elle, se consacre plus spécifiquement à la question des relations, dialogues et interactions entre divers médiums et techniques dans les arts (tant visuels que littéraires), avec un souci théorique évident. Elle constitue une source stimulante pour la recherche interdisciplinaire. Autrement, parmi les ouvrages qui touchent directement aux questions de l'interdisciplinarité et de la scène contemporaine interartistique, je retiendrai, pour le propos de cette note de lecture, deux livres publiés dans le contexte de recherches en arts visuels et en histoire de l'art contemporain, et deux portant sur des aspects de la scène contemporaine. J'ai pris le parti de retenir les ouvrages qui tenaient compte de la question interdisciplinaire rattachée, de près ou de loin, à celle de la scène contemporaine.

\section{L'INTERDISCIPLINARITÉ EN QUESTION}

J'ouvrirai cette traversée de lectures en commentant, de façon non exhaustive, deux ouvrages qui n'ont a priori rien à voir avec le sujet des arts de la scène contemporains, mais qui constituent, du moins à ma connaissance, les deux seuls livres publiés au Québec sur la question interdisciplinaire: Penser l'indiscipline. Recherches interdisciplinaires en art contemporain ${ }^{4}$ et L'espace traversé. Réflexions sur les pratiques interdisciplinaires en art ${ }^{5}$.

Il m'a paru important d'en rendre compte, même si cette réflexion a comme ancrage la pratique des arts visuels (incluant la performance), étant donné que l'histoire de l'art contemporain semble avoir l'initiative de la

$$
+4 *
$$

2. Voir, par exemple, Marie-Chrısune LESAGE, "Le theâtre ec les autres arts: matériaux composites", Dominique LAFON [dir.], Le thétatre quebecosis, 1975-1995, Ottawa, Fides, 2001, p 335-360. Ce rexce se voulait une réflexion synthétique sur l'orıgıne du décloisonnement artistique sur la scène québécoise (questionnement des codes théâtraux et influence de la performance: Opéra-Fête, Zoopsie), puis sur les créations plus récentes qui croisent le thêatre avec la danse ou avec des arts visuels comme l'ınstallation er les nouveaux médias (de Carbone 14 à Ex Machına et au Thêatre Ubu).

3. La revue Les Cahiers de thếtre Jeu a notamment publié un dossiêr "Théâtre et musiquè ( $\left.{ }^{\circ} 124,2007\right)$ et un numéro consacré aux a Paysages du corps $\left(^{\circ} 125,2008\right)$. Cependant, ce périodique culturel n'a pas pour objectıf d'offrir une réflexion théorique sur ces questions. La revue universitaire L'Annuaire théatral a quant à elle consacré plusieurs dossiers à des sujets interdisciplinaires: "Theâtre, musique et environnement sonore" ( $\mathrm{n}^{\circ} 25$, printemps 1999), * Theâtre et ınterdisciplinarité" ( $n^{\circ} 26$, automne 1999), "Encre thêatre et cınéma... " ( $n^{\circ} 30$, automne 2001), "Cirque et théâtralıté: nouvelles pistes $*\left(n^{\circ} 32\right.$, automne 2002$)$.

4. Lynn HUGUES et Marie-José LAFORTUNE [dir.], Penser lindisciplene. Recherches interdisctplinaires en art contemporain/Creative Confwsions. Interdisciplenary Practuces in Contemporary Art, Montreal, Optica, 2001.

5. Guy LaRamée [dir.], L'espace traverse. Reflexions sur les pratiques interdssciplenatres en art, Trois-Rivières, Editions d'art Le Sabord, 2001. 
réflexion sur l'interdisciplinarité. Les deux sont des ouvrages collectifs publiés en 2001 et font suite à des colloques organisés sur la question; ils présentent des textes à la fois de théoriciens et d'artistes, qui témoignent de leur pratique interartistique ${ }^{6}$. Plutôt que de les traiter séparément, je vais mettre en parallèle les réflexions ouvertes par certains auteurs concernant le contexte historique de la notion, son appropriation par les pratiques artistiques et la réflexion théorique qui en émerge.

Dans leur introduction à Penser lindiscipline, Lynn Hugues et Marie-Josée Lafortune rappellent qu'il existe très peu d'ouvrages qui débattent de la notion d'interdisciplinarité dans les arts, tant au Québec qu'au Canada, hormis quelques numéros de revues spécialisées. Le choix qu'elles font de parler d'«indiscipline" plutôt que d'interdisciplinarité a été inspiré, comme elles l'expliquent, par la réflexion menée par W. J. T. Mitchell dans un texte intitulé "Interdisciplinarity and Visual Culture" ${ }^{7}$, où l'auteur affirme que l'interdisciplinarité toucherait davantage au problème de la compétence professionnelle à l'intérieur d'un champ spécifique, en opérant une fracture qui remet en question une pratique disciplinaire fondée sur la continuité d'un apprentissage technique, social, professionnel. L'indiscipline, quant à elle, mettrait en place des formes de turbulence ou d'incohérence aux frontières internes et externes des disciplines (p. 11). Cela dit, l'indiscipline fait partie des stratégies interdisciplinaires dans le contexte de la création artistique.

Parler d'interdisciplinarité ne peut se faire sans qu'on interroge ce qui est ainsi mis en interrelation, soit les disciplines. Tim Clark, dans un texte intitulé "Disciplina and Disciplinary Research" ${ }^{8}$, rappelle que le mot disciplina a ses racines dans l'Antiquité grecque et qu'il se rapportait alors à tout ce qui était enseigné (instruction et apprentissage), en référence aux circonstances de la vie, tout comme à la science, aux arts, à la morale et à la politique (p. 75), mais selon une unité des connaissances. Ce concept de discipline a été transformé radicalement au XVIII ${ }^{e}$ siècle, avec la constitution de chaque discipline en son sens moderne, qui impliquait alors qu'elles possèdent une cohérence théorique et méthodologique propre, intrinsèque à chacune. Au XIX siècle, les disciplines ont intériorisé cet objectif et elles sont devenues de plus en plus spécialisées, jusqu’à être rigidement comparti-

$$
+\div
$$

6. Les textes sont, dans les deux livres, écrits en français ou en anglass et suivis ou précédés d'une traduction qui en faut le résumé.

7. W. J. T. MITCHELL, «Interdisciplinarıty and Visual Culture", The Art Bulletrn, decembre 1995

8. Tım ClARK, "Disciplına and Disciplinary Research", Lynn HUGUES er Marie-josée LAFORTUNE [dir.], op ctt, p. $72-85$. 
mentées en regard des principes les gouvernant (p. 78). Les disciplines, qu'elles soient scientifiques, sociologiques ou artistiques, sont devenues tout au long du XX⿳亠口冋口位le de plus en plus subdivisées, ce qui a généré une critique de la limitation, d'où est issue l'interdisciplinarité. Les tenants de l'interdisciplinarité mettent en question la rigidité disciplinaire, son cloisonnement, ainsi que l'apprentissage fragmenté qui menacerait la liberté et l'invention (p. 83).

C'est aussi ce que rappelle Ron Burnett dans son texte "Disciplines in Crisis" ", en expliquant que la crise des disciplines académiques et artistiques est reliée aux transformations radicales des conditions sociales et culturelles de communication, de connaissance, d'information et de création (p. 32). Souvent, explique l'auteur, on tient pour acquis que la technologie est la cause principale de ce changement, mais il est annoncé depuis la venue des systèmes de communication de masse. Son point de vue défend une approche transdisciplinaire qui ne doit pas être confondue avec l'interdisciplinarité ou la multidisciplinarité, qui, elles, restent liées au cadre de la recherche disciplinaire classique. Son contenu et sa méthodologie se situent au nexus d'une variété d'approches. C'est un complexe entre-deux au sein de stratégies pour l'étude et la compréhension de l'action humaine et des phénomènes culturels, scientifiques et technologiques (p. 35). Cette approche transdisciplinaire est marquée par la pensée d'Edgar Morin, dont les travaux ont eu une influence importante dans la seconde moitié du XXe siècle. Selon Burnett, Morin propose de développer des stratégies, méthodes et pratiques qui vont transformer les liens entre les disciplines en de véritables connexions, lesquelles incluent celles entre l'art et la théorie. Cette vue holistique, qui réunirait les arts et la science au-delà de leurs différences et dans un but commun, est parfois réinterprétée dans le sens d'une «vision utopique" rattachée à la question de l'interdisciplinarité; du moins, c'est ce qui ressort de quelques textes publiés dans L'espace traversé. Si le propos de Burnett demeure d'une grande rigueur (il discute à fond d'un aspect méthodologique de la recherche interdisciplinaire), d'autres tendent, me semblet-il, à tout mélanger sous cette bannière: les arts, la mondialisation, l'écologie, la science, les identités culturelles, etc. ${ }^{10}$; - et ce, peut-être dans la

$$
+4+
$$

9. Ron BurnetT, "Disciplines in Crisis», Guy LARAMÉE [dir.], op cit., p. 32-43.

10. Le texce de Danielle BOUTET, "À la recherche d'un nouveau projet artiscique" (Guy LARAMÉE [dir.], op. cit., p. 44-54) me paraît caractérıstıque de cette vısion utopique de l'interdisciplinarité; et on lira avec profic la réponse à ce rexte faite par le cinéaste Pierre Hébert ("Multidısciplinarité ec hıstoricité», Guy LARAMÉE [dir.], op. ctt. p. 136-145), qui questionne vivement les cenants et aboutissants de cette position. 
foulée des performance studies américaines (ici récupérées de façon plutôt libre et subjective), qui recourent au terme interdisciplinaire pour nommer des pratiques théoriques et politiques déplaçant les subjectivités pour en faire apparaître de nouvelles. Andreas Kahre ${ }^{11}$ identifie clairement cette vision utopique rattachée à l'inter- et à la transdisciplinarité: le modèle utopique, écrit l'auteur, tente de remplacer un ensemble fracturé de méthodologies scientifiques et artistiques par une métadiscipline intégrante, dans une vision mondiale. Ce modèle postule que les pensées scientifique, écologique et artistique doivent être reformulées en une pratique holistique (p. 128). En ce sens, poursuit-il, l'interdisciplinarité n'a pas de forme précise et ne peut être une discipline, seulement une approche, peut-être une méthode.

Dans un texte d'une très grande limpidité, Patrice Loubier ${ }^{12}$ tente une définition de l'interdisciplinarité dans les arts visuels contemporains en rappelant, dans un premier temps, que le découpage disciplinaire (en médiums distincts et selon la maîtrise d'un savoir-faire technique et esthétique) est intimement lié à la dynamique de la modernité, "[...] c'est-à-dire à ce double processus d'autonomisation de l'art lui-même comme sphère distincte de la praxis et de différenciation interne des arts en médiums analogues à autant de compétences spécialisées" (p. 29). En effet, le mot discipline renvoie à la fois à la clôture de chaque forme artistique - qui, dans la période moderne, a été un signe d'émancipation - et aux règles qui les particularisent. Chaque art devait éliminer, autant que possible, tout élément susceptible d'être emprunté à un autre art. Selon l'auteur,

[s]i l'art moderne a été disciplinaire, l'art contemporain générique, lui, est par avance méta-, voire postdisciplinaire: la création ne s'ancre plus d'emblée à la tradition d'un médium donné, voire n'implique plus la maîtrise d'un savoir-faire plastique, mais se déploie plutôt à partir d'un espace qui se situe entre les disciplines plutôt qu'à l'intérieur d'elles, les artistes puisant indifféremment selon les exigences de leurs projets aux médiums spécifiques comme à de multiples techniques ou protocoles issus de secteurs d'activité non artistiques (p. 25).

C'est là la position défendue par l'auteur, qui prend tout de même la peine de préciser qu'il existe deux formes d'interdisciplinarité artistique: une première (au sens restreint) où la création d'une œuvre résulte de la

$$
++
$$

11. Andreas KAHRE, "The Interdisciplinary Condition ", Guy LARAMEE [dir.], op. cit., p. 126-135.

12. Patrice LOUBIER, "Du moderne au contemporain. Deux versions de l'interdisciplinaritén, Lynn HuguES et MarieJosée LAFORTUNE [dir.], op. cit, p. 22-29. 
circulation et du dialogue entre deux ou plusieurs disciplines; et une seconde (au sens élargi), où le travail s'inscrit d'emblée entre les disciplines et à la rigueur hors d'elles (p. 26). Dans la seconde acception, "on postule une zone indifférenciée, quelque chose comme un tissu conjonctif, un "entredisciplines" où l'œuvre sera définie [...] par la seule généricité de son appartenance à la pratique art" (p. 26). Aussi séduisante que soit cette hypothèse, elle soulève toutefois plusieurs questions: qu'est-ce que l'art en général sans les arts qui en déterminent le système ? Comme l'affirme très pertinemment l'artiste Louise Prescott ${ }^{13}$, " l'artiste interdisciplinaire ne peut pas, ne doit pas se passer des recherches fondamentales en tant que disciplines: c'est là qu'il va puiser ses savoirs et qu'il peut les appliquer" (p. 109). Et les traditions disciplinaires sont toujours réinterprétées de génération en génération. La question qui agite artistes et théoriciens consiste au fond à savoir si l'interdisciplinarité est une nouvelle catégorie esthétique au sein de laquelle le découpage des disciplines entre elles serait obsolète, ou si elle est avant tout une pratique, une méthode de création au moyen de techniques et de médiums artistiques multiples. Je pencherais plutôt, à l'instar de Louise Prescott et d'Andreas Kahre, pour la seconde hypothèse, sans écarter le fair que la pratique interdisciplinaire puisse faire émerger de nouvelles formes artistiques (l'installation en est un exemple, tout comme les arts médiatiques). Pour Kahre, les disciplines existent dans un paysage commun et incluent la création de différents cadres de référence, comme des cartes ou des systèmes de projection qui accentuent certaines caractéristiques, se détournent d'autres, et ainsi produisent des topologies qui peuvent être réclamées comme territoires (p. 132).

Aussi me parait-il intéressant de penser l'interdisciplinarité artistique comme une pratique, une méthode de création caractéristique des arts contemporains (mais pas uniquement: l'avant-garde pratiquait aussi cette forme de décloisonnement des arts, bien que les enjeux fussent radicalement différents) qui, si elle met à mal les frontières entre les arts, ne les élimine pas complètement - autrement, la dynamique de l' "inter ${ }^{14}$ " disparaîtrait. C'est là l'hypothèse de la transdisciplinarité. Pourtant, dans les créations artistiques interdisciplinaires, c'est justement cette dynamique de l'«inter" qui permet de remettre en jeu, en les déplaçant, les forces créatrices et les langages

$$
+\div
$$

13. Louise PRESCOTT, «La pratique de l'arr ou le complexe d'Ulysse», Guy LARAMEE [dir.], op. cat., p. 102-115. 14. Pour une discussion sur le sens de a inter», voir Éric MÉCHOULAN, aIntermédialités: le temps des illusions perduesn, Intermédalités, $n^{\circ} 1$, printemps 2003 , p. 9-27. 
artistiques. L'artiste Vera Frenkel ${ }^{15}$ écrit avec justesse que l'interdisciplinarité est le lieu du doute et de l'incertitude au sein du processus de création, et qu'il importe de préserver cette inquiétude vitale (p. 38). Elle identifie ensuite trois approches possibles de l'interdisciplinarité, que l'on pourrait résumer de la façon suivante: la première crée une sorte de feuilleté où les langages se font écho afin de créer un tout harmonieux; la deuxième travaille à confronter les cadres de référence des arts, dans le sens d'une intégration perceptive; et la troisième relèverait de la pratique du métissage, en tentant d'inventer sa propre grammaire et de créer des ponts entre des langages artistiques différents qui ne peuvent être aisément mis en relation (p. 41). On peut penser qu'il existe en fait autant de processus interdisciplinaires que de dialogues, de collaborations et de rencontres aux frontières des différents territoires artistiques.

En somme, ces deux ouvrages collectifs ont le mérite d'aborder de front la question des recherches interdisciplinaires en art, du point de vue tant théorique qu'artistique; et, si les textes sont d'un intérêt inégal, on en retient tout de même quelques réflexions essentielles qui se révèlent pertinentes pour les arts de la scène, où l'on retrouve aussi cette dynamique interartistique.

\section{LES ARTS DE LA SCËNE ET L'INTERARTISTIQUE}

Il est étonnant de constater que peu ou pas d'ouvrages traitent de la question de l'interartistique sur la scène contemporaine. Depuis plus d'une trentaine d'années au Québec, les arts de la scène (théâtre, danse, cirque, musique, performance) opèrent différents types de croisements, voire d'hybridations, qui ont donné lieu à des formes scéniques inédites relevant certainement de l'interartistique. Pour d'aucuns, c'est dans l'avènement de la performance qu'il faut chercher l'origine de ce décloisonnement disciplinaire: elle est en effet considérée comme une pratique au carrefour d'arts aussi diversifiés que la danse, le théâtre, la musique, la peinture, la sculpture ou l'architecture. Si la performance a pu ouvrir la porte à des processus de déterritorialisation et de déplacement entre les arts, elle demeure une pratique qui se veut "adisciplinaire" et dont les fondements ${ }^{16}$ sont clairement distincts des formes interartistiques telles qu'on les trouve sur la scène contemporaine. On pourra

$$
+4
$$

15. Vera FrenKel, "A Kind of Listening. Notes From an Interdisciplinary Practice», Lynn Hugues et Marie-Josee LAFORTUNE [dir.], op. cit., p. 31-47.

16. Sur la performance comme art d'action, je renvoie au livre collectuf Arts diattitudes. Discusston, actzon, interaction (Ruchard MARTEl. [dır.], Québec, Inter Édıreur, 2002), qui rassemble les interventions du colloque tenu à Québec en septembre 2001. 
se reporter au livre collectif intitulé Théâtralité, écriture et mise en scène ${ }^{17}$, qui, s'il n'est pas récent, pose entre autres les bases d'une réflexion nécessaire sur les différences entre l'art de performance et l'art théâtral. Si au départ la performance s'oppose au théâtre et à ses codes mimétiques de représentation, la pratique de certaines compagnies québécoises (L'Eskabel, Théâtre Zoopsie et les débuts de Carbone 14) a pu se situer entre les deux arts, l'esprit de la performance venant vivifier le théâtre. Ainsi, comme l'écrit Philip Monk ${ }^{18}$, on peut se questionner sur l'usage que fait le théâtre de la performance et, inversement, sur ce que la performance de l'artiste révèle de la théâtralité (p. 114). De même Josette Féral ${ }^{19}$ explore-t-elle dans son texte ce qui caractérise la performance (manipulation du corps du performeur, manipulation de l'espace et relation entre l'artiste et les spectateurs) et ce qui la distingue du processus de la théâtralité. Elle en arrive toutefois à une conclusion intéressante: la thêatralité serait faite de deux ensembles différents, l'un (performatif) enraciné dans les réalités de l'imaginaire (qui ont leur origine dans le sujet et laissent parler des flux de désir), l’autre (théâtral) fondé sur des structures symboliques précises (qui inscrivent le sujet dans la loi et les codes, c'est-à-dire dans le symbolique). «Du jeu de ces deux réalités naît la théâtralité" (p. 137). Ainsi parlera-t-elle, pour l'acte performatif, d'«infrathéâtralité". Cette sorte d'infra-thêâtralité réapparaît dans certaines créations scéniques actuelles, sans qu'il s'agisse d'actes performatifs au sens propre du terme. Certaines interactions entre la danse et le théâtre, par exemple, ou encore entre le théâtre et les techniques de l'image tendent à faire émerger une théâtralité déplacée dans ses composantes, en retrait par rapport au symbolique et au sujet, esquissant de nouvelles façons de représenter l'imaginaire en scène.

S'il est utile de se référer à cet ouvrage pour se remettre en mémoire que les premières explorations interdisciplinaires sont advenues avec l'art de la performance, plus près de nous, on retrouve essentiellement deux livres qui touchent aux questions de la scène contemporaine et de l'interartistique: Théâtre, multidisciplinarité et multiculturalisme $e^{20}$ et La face cachée du théatre de l'image ${ }^{21}$, publiés par Chantal Hébert et Irène Perelli-Contos. Leurs

$$
++\div
$$

17. Josette FÉRAL [dir.], Theâtralité, écriture et mise en scène, Montréal, Huttubise HMH, 1985.

18. Philip MONK, "Véhicule commun: arcistes en performance”, Josetre FÉRAL [dir.], op. cit., p 113-123.

19. Joserte FÊraL, " Performance et theârralité: le sujet démystifié, Josette Féral [dir.], op. cit., p. 125-140.

20. Chancal HÉBERT et Irène PERELu-CONTOS [dir.], Theatre, multzdiscaplinarité et multiculturalsme, Québec, Nuıt Blanche Éditeur, 1997.

21. Chantal Hébert et lrène Perelul-Contos, La face cachée du theatre de limage, Québec/Paris, Les Presses de l'Unversité Laval/L'Harmattan, 2001. 
recherches sont centrées sur le thêâtre, mais les auteures ont développé un regard ouvert sur les autres arts et, surtout, une approche théorique interdisciplinaire. Le premier ouvrage constitue les actes d'un colloque qui s'est tenu en juin 1994, à Québec, et qui avait comme objectif d' «interroger les processus et les fondements de la création théâtrale actuelle de même que ses relations avec le public et les autres domaines artistiques ${ }^{22}$ ». Le choix du préfixe multi, dans le titre, n'est pas discuté par les auteures, qui affirment simplement ne pas avoir voulu engager une polémique autour des préfixes possibles (multi, pluri, inter, trans), préférant examiner un pan de la réalité théâtrale actuelle. Le texte de Rodrigue Villeneuve, cependant, propose une réflexion autour de la notion d'interdisciplinarité, qu'il distingue de la multi- ou de la pluridisciplinarité, "[qui] est un fait de juxtaposition: une recherche ou une manifestation multidisciplinaire réunit, met ensemble, à propos d'un même objet, des disciplines qui, par delà les effets d'une certaine contamination, conservent chacune leur spécificité2 ${ }^{23}$. L'interdisciplinarité serait plus exigeante, car elle imposerait aux disciplines réunies une finalité commune et elle donnerait naissance à un processus qui transcende les disciplines de départ, ce qui se rapproche de la transdisciplinarité telle que discutée précédemment.

Lauteur explore ensuite la question suivante: le théâtre est-il un art interdisciplinaire? En retournant à un texte de Walter Gropius, du Bauhaus, qui prend pour modèle interdisciplinaire la forme théatrale, il récuse l'idée, affirmant que le théâtre est bien une discipline. Autre modèle historique examiné: celui du Gesamtkunstwerk de Wagner, figure incontournable lorsqu'on évoque la relation entre les arts sur la scène. Et c'est avec justesse qu'il précise que l'œuvre d'art totale n'a de totalisante que le nom, car Wagner, dans ses écrits, parle moins de fusion que de nouvel alliage entre les arts frères que sont la musique, le drame (ou la poésie) et la danse, auxquels s'ajoutent (mais dans une moindre mesure) l'architecture, la peinture et tout ce qui relève des arts de l'espace. Le rêve wagnérien n'en est pas un de fusion mais d'addition dans le respect des particularités disciplinaires. "Mais alors que le Bauhaus, croyant faire du théâtre, propose dans les faits un véritable embryon de manifestation interdisciplinaire, Wagner, lui, en se réclamant de ce qu'on définirait aujourd'hui comme l'interdisciplinarité, est incapable

$$
+4
$$

22. Yann Rousset, "Présentation", Chancal HêBert et Irène Perelli-CONTos [dir.], Théàre, multidiscaplinarité et multrculturalsme, p. 8.

23. Rodrigue VILLENEUVE, «Le thêatre, un art interdısciplinaire? ", Chantal HÉBERT et Irène Perel.l-Contos [dir ], Theatre, multidsctplinarté es multiculturalisme, p. 162. 
d'échapper au théâtre.» (p. 165) C'est surtout à la forme opératique, fondée sur le drame et la musique, qu'il n'échappe pas. La réflexion de Villeneuve permet de clarifier une chose concernant l'interartistique: telle qu'on la retrouve sur les scènes contemporaines, elle n'est pas la résurgence ou un avatar du Gesamtkunstwerk, mais elle ne correspond pas non plus à cette synthèse "au-delà des disciplines artistiques" proposée comme définition de l'interdisciplinarité. La référence au Bauhaus apparaît plus pertinente pour penser les origines avant-gardistes de l'interdisciplinarité, mais l'auteur distingue ce qu'il nomme l'" esthétique interdisciplinaire" de l'art du théâtre, ce qui à mon sens pose problème, car, comme cela a été discuté plus haut, l'interdisciplinarité serait moins une esthétique qu'une praxis. Ce processus de création prend souvent comme base un ou plusieurs champs disciplinaires qui vont être mis en interaction selon les configurations les plus diverses (qui dépendent du projet initial); ainsi, l'art interdisciplinaire se trame à partir des disciplines, qu'il entrecroise et met en tension afin de construire une œuvre qui sera métissée de différents arts agglomérés, juxtaposés, en dialogue ou encore réinventés. Il n'y a pas d'esthétique interdisciplinaire (encore moins de genre !), mais des pratiques nouvelles qui ouvrent sur des projets diversifiés et qui, le cas échéant, peuvent fonder de nouveaux territoires artistiques.

L'ouvrage collectif présente, par ailleurs, une variété de textes qui réfléchissent tant à la pratique scénique qu'à la question de l'altérité au théâtre (sous le chapeau du multiculturalisme) et à la place du texte dans le théâtre actuel. Il est intéressant de noter que deux textes portent sur le travail de Carbone 14, qui fut, à une certaine époque, emblématique d'un thêâtre interartistique. La contribution de Roger Chamberland, «L'expérience du chaos et la pragmatique du corps ${ }^{24}$ ", est particulièrement intéressante parce qu'elle s'inspire de nouvelles théories scientifiques (la théorie du chaos) pour analyser l'écriture scénique éclatée du spectacle Peau, chair et os de Carbone 14. De même, la réflexion de Chantal Hébert, «De la mimesis à la mixis ou les jeux analogiques du théâtre actuel ${ }^{25}$ ", porte sur le travail de Robert Lepage, dans une perspective elle aussi inspirée par les chercheurs de la scienza nuova, "qui est à la jonction de deux cultures, la culture scientifique et la culture humaniste, et qui cherche à saisir l'intelligibilité de l'univers à l'aide d'instruments conceptuels inédits, s'exprimant entre autres par les termes "hasard organisateur", "chaos entropique" [...]" (p. 26). On retrouve

$$
+4+
$$

24. Roger Chamberland, a L'expérience du chaos et la pragmatique du corps», Chantal Hebert et Irène Perel.uCONTOS [dir.], Theâtre, multidusciplenartí et multrculturalisme, p. 13-23.

25. Chancal HÉBERT, " De la mimesis à la mixus ou les jeux analogiques du thétre actuel ", ibid., p. 29-39. 
en effet, souvent associées aux analyses d'œuvres interartistiques, des tentatives de renouvellement conceptuel pour aborder ces créations qui paraissent mettre en place de nouvelles dynamiques de représentation. Les théories du chaos, celle de la complexité développée par Edgar Morin ainsi que les approches cognitives sont privilégiées dans nombre d'études ${ }^{26}$. Ainsi les créations interartistiques appellent-elles des approches interdisciplinaires, c'est-à-dire une nouvelle théorie critique permettant de mieux saisir les enjeux de l'écriture scénique actuelle, qui mêle les arts et les techniques. La raison pour laquelle, selon les auteurs, ces théories paraissent fertiles pour l'étude de la scène contemporaine réside dans une hypothèse qu'ils semblent partager: ce thêâtre visuel pourrait être la manifestation de nos modes de perception et il participerait "à l'invention d'une écriture dramatique et scénique qui s'accorde on ne peut plus harmonieusement à la civilisation de l'audiovisuel et des nouvelles technologies dans laquelle nous vivons" (p. 26). L'image et certains de ses procédés s'apparentent analogiquement à certaines expériences perceptives et à divers phénomènes physiques que les sciences de la complexité tentent d'expliquer. Si ces approches sont d'un intérêt certain, il est étonnant de retrouver une seule étude qui fasse appel aux esthétiques des autres arts: le texte de Frank Popper intitulé "Les arts plastiques et les nouvelles technologies: affinités avec l'art théâtral ${ }^{27}$ " trace un panorama d'œuvres plastiques qui présentent de telles caractéristiques, sans en approfondir la dynamique proprement interartistique. Par ailleurs, un seul livre à ma connaissance adopte un point de vue esthétique interdisciplinaire, et c'est celui de Michèle Febvre, Danse contemporaine et théâtralitée ${ }^{28}$, dans lequel l'auteure approfondit la notion de théâtralité pour penser un pan de la danse contemporaine qui est empreint d'une certaine théâtralité. De façon générale, c'est l'image, l'éclatement des formes et ses rapports avec les technologies en scène qui retiennent l'attention des chercheurs, et cet aspect visuel de la scène contemporaine mis en relation avec les nouvelles techniques oriente la réflexion vers des approches de type cognitiviste.

$$
+\div+
$$

26. Dans l'ouvrage Penser l'ndiscrpline, dıscuté plus tôt, on retrouve aussı un excellent texte quı recourt aux sciences cognitives pour analyser une ceuvre d'installation médiatique de Douglas Gordon: Christine Ross, «Inhibicions perceptıves. Ce que l'art contemporan a à dıre aux sciences cognıtivesn, Lynn HUGUES et Marie-Josée LAFORTUNE [dir.], op ctt., p. 179-187.

27. Frank POPPER, «les arts plastıques er les nouvelles technologıes: affinutés avec l'art thêâtral ", Chantal HÉBERT et lrène PERELLI-CONTOS [dır.], Théatre, multidisciplinarité et multzculturaltsme, p. 171-183.

28. Michèle FEBVRE, Danse contemporaine et theâtralité, Paris, Éditions Chrion, 1995. L'auteure vit à Montréal et a été directrice du Département de danse de l'UQAM, maıs le livre a été publıé en France. Il traite toutefois de nombreuses créations de chorégraphes québécois. 


\section{COMME UN «FEUILLETÉ D'IMAGES» ET DE PENSÉES}

Je terminerai cette traversée de lectures par le livre La face cachée du théatre de limage, qui possède plusieurs qualités, dont celle de présenter une étude qui, si elle prend appui sur l'œuvre de Robert Lepage (emblématique d'une scène à la fois visuelle et interartistique), a toutefois une portée théorique beaucoup plus générale: on y développe une approche proprement interdisciplinaire inspirée de celle du père de l'interdisciplinarité théorique, Edgar Morin, approche qui pourrait aisément servir à l'analyse d'un pan des créations scéniques actuelles. L'autre qualité de cette étude (outre le fait de ne pas être un ouvrage collectif: les collectifs n'offrent jamais une réflexion construite autour d'un axe théorique et esthétique précis) est d'allier à l'interdisciplinarité théorique une approche qui croise aussi les théories esthétiques, ce qui manquait jusqu'à présent aux publications sur le sujet.

Le livre est une théorisation des enjeux cachés du thêatre de l'image, et plus particulièrement de celui créé par Robert Lepage. Mais il s'agit moins d'un ouvrage sur l'esthétique de ce metteur en scène que sur les transformations "dans notre manière de regarder, de nous représenter et donc de connaître ce qui nous entoure, à un moment précisément où s'implante un autre ordre visuel, largement tributaire de ce qu'on appelle les "technologies de l'image" " (p. 12). Le théâtre de Lepage sert d'appui exemplaire pour expliciter cette dynamique de la scène contemporaine qui ouvre, selon les auteures, à un théâtre de la connaissance. Dans la première partie, intitulée "Théâtre et complexité», sont exposés les prémisses esthétiques et les fondements théoriques de la réflexion, qui vont ensuite servir d'assises aux analyses déployées dans les deux parties suivantes. D'une part, l'écriture scénique qui fonde le théâtre de l'image, et plus particulièrement celui de Lepage, est définie comme "[...] un assemblage, un brassage ou un bricolage d'objets, de paroles, de musiques, de sons, d'éclairages, de textes, de gestes, de mouvements, d'appareils technologiques, d'écrans, etc. [...]" (p. 9), et ce langage "[...] consiste en des images multidimensionnelles qui, en suscitant des correspondances et des associations visuelles illimitées, donne la "parole" au regard spectateur" (p. 11) - ce qui caractérise nombre de créations de la scène contemporaine, qu'elles soient issues du théâtre, de la danse, du cirque ou de performances technologiques. D'autre part, la notion de complexité, telle que théorisée par Edgar Morin, est ici reprise et présentée comme concept central permettant d'ouvrir et de saisir la dynamique du théâtre de l'image. On en présente les trois principes clés: dialogique, hologrammatique et récursif, mais toujours en effectuant un pont avec le théâtre de Lepage, de 
manière à souligner la pertinence des notions pour comprendre la scène contemporaine.

La seconde partie met en pratique cette approche, afin de montrer comment ce thêâtre expérimente visuellement la mouvance de la pensée et de la connaissance par rapport au monde. Une seule œuvre de Lepage sert de modèle opératoire: Vinci (1988), son premier solo, qui recourt à des images de fabrication plutôt artisanale, loin des techniques sophistiquées qui feront ensuite sa marque. L'étude de Vinci sert en fait de tremplin à la mise en place d'un nouveau cadre épistémologique à partir duquel penser la scène contemporaine. La force de ce chapitre est d'équilibrer parfaitement la réflexion abstraite et concrète, les scènes analysées permettant non seulement de valider les prémisses théoriques mais aussi de montrer comment ce théâtre opère déjà selon la logique de la complexité, refondant ainsi une certaine pensée sur la représentation. Selon les auteures,

[à] la différence de la représentation thêâtrale traditionnelle [...], les représentations du théâtre de limage sont avant tout des manipulations de matériaux ou d'éléments (objets, textes, images, sons), fondées non pas sur le réel, mais sur les modèles ou constructions mentales que nous nous formons de la réalité (p. 56).

Les aventures visuelles proposées par ce théâtre mettent en réseau des textures visuelles et sonores de façon non logique et linéaire, mais plutôt associative et analogique (p. 45). Il manifeste ainsi son intention de "faire partager un réseau de sensations actualisé dans le visible et inscrit dans le sensible" (p. 48), de façon à ce que le sujet (acteur ou spectateur) entre dans le tableau et soit au centre de l'image. L'étude insiste sur le principe du «mixage visuel ", terme emprunté à l'art vidéographique, afin de montrer comment le thêatre de l'image interroge le regard du spectateur, en l'invitant à voir autrement et à devenir conscient de son rôle dans la constitution des images globales. Cette partie a retenu plus particulièrement mon attention, car elle analyse la composition des images dans Vinci à la lumière du langage de l'image vidéographique. Cette étude, qui associe analyse de l'image scénique, théorie de la complexité et esthétique vidéographique, se révèle très fertile pour la compréhension de la dynamique visuelle à l'œuvre sur la scène, sans que cette dynamique fasse usage de la vidéo: c'est analogiquement ou comme mode de composition que sont repris les principes vidéographiques de surimpression, d'incrustation ou encore de jeux de volets dans les scènes discutées. Il s'en dégage des effets de dislocation, qui tendent à faire "éclater l'illusion référentielle" (p. 80), de multiplication des points de vue en simultané ou de 
"feuilleté d'images" (p. 104), qui, entre autres procédés, créent un théâtre visuel qui semble avoir "la faculté de donner à voir les assises et les mécanismes qui régissent nos modes de perception et nos processus cognitifs" (p. 79). Une réflexion semblable est menée autour de la photographie et de la musique, ce qui permet de souligner l'aspect implicitement interartistique du théâtre de l'image, ouvert sur la dynamique expressive des autres arts.

Le dernier chapitre, intitulé "Théâtre et connaissance», effectue un retour plus théorique sur les hypothèses et analogies filées tout au long de la deuxième partie. Déplaçant d'abord le regard vers une analyse étymologique très précise, les auteures rappellent l'importance du "regard spectateur" inscrit dans les racines qui composent le mot théâtron et tous ses dérivés. L'analyse est passionnante ainsi que les conclusions auxquelles elles arrivent: "Cela fait du spectateur (théôros) un sujet regardant dont l'activité visuelle consiste, comme l'écrivait Aristote dans la Poétique (1148b 16), à "apprendre et penser en regardant" plutôt qu'à voir passivement." (p. 148) Aussi ce théâtre de l'image travaillerait-il à la transformation du regard devant les changements perceptifs qui sont les nôtres, amenant notamment le spectateur à prendre conscience de "comment il voit", ce que Morin désigne comme étant le "quatrième regard" (p. 154). Ce "théâtre d'opérations", pris ici au sens propre (les "problèmes de vision" que nous éprouvons devant l'avènement de la nouvelle culture technologique) et au sens figuré (une machine à jouer), compose une vision du monde fondée sur des "immatériaux" (p. 164), emblématique de la transformation de la pensée et du regard que nous vivons. Cette dernière partie s'appuie sur des exemples tirés de l'ensemble de l'œuvre de Lepage, mais cette fois en posant un regard sur le regard, tel qu'il a été approfondi dans le second chapitre avec Vinci; autrement dit, la réflexion est recadrée autour de la question de la connaissance modélisée par la scène imagée.

Cet ouvrage est le seul à ma connaissance qui propose une véritable théorisation des transformations observées sur la scène contemporaine, conceptualisation fortement inspirée par la pensée interdisciplinaire d'Edgar Morin. On pourra avoir certaines réserves par rapport à l'importance magistrale accordée au caractère cognitif du théâtre de l'image, mais il n'en demeure pas moins que les auteures ouvrent la voie à une autre façon de penser la scène actuelle, tout en ménageant un espace aux aspects interdisciplinaires, qui sont traités avec une grande justesse théorique. On peut regretter que seule l'œuvre de Robert Lepage ait servi d'appui à cette réflexion théorique: la pensée des auteures aurait peut-être gagné à s'exercer sur d'autres créations scéniques, de manière à démontrer toute la portée de leurs hypothèses. D'un 
autre côté, en se concentrant sur les créations de Lepage, elles développent un regard qui est approfondi avec une grande précision. Étant donné la profusion de représentations scéniques qui échappent de plus en plus aux formes établies, il est étonnant de ne pas trouver davantage de publications qui se penchent sur ces nouvelles scènes qui convoquent librement tous les langages artistiques. Les ouvrages collectifs, s'ils ont l'avantage de défricher le terrain, n'offrent pas la profondeur et la cohérence d'une pensée qui prend le risque de se confronter aux formes contemporaines et de proposer de nouvelles voies d'accès. C'est là tout le mérite du livre de Hébert et Perelli-Contos. 